\title{
Fate of Artificial Sweeteners in Wastewater Treatment Plants in New York State, U.S.A.
}

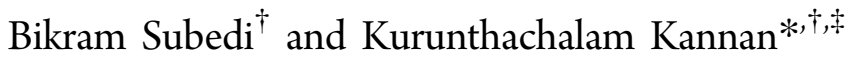 \\ ${ }^{\dagger}$ Wadsworth Center, New York State Department of Health, and Department of Environmental Health Sciences, School of Public \\ Health, State University of New York at Albany, Empire State Plaza, P.O. Box 509, Albany, New York 12201-0509, United States \\ ${ }^{\ddagger}$ Biochemistry Department, Faculty of Science and Experimental Biochemistry Unit, King Fahd Medical Research Center, King \\ Abdulaziz University, Jeddah 21589, Saudi Arabia
}

ABSTRACT: Very few studies describe the fate of artificial sweeteners (ASWs) in wastewater treatment plants (WWTPs). In this study, mass loadings, removal efficiencies, and environmental emission of sucralose, saccharin, aspartame, and acesulfame were determined based on the concentrations measured in wastewater influent, primary effluent, effluent, suspended particulate matter (SPM), and sludge collected from two WWTPs in the Albany area of New York State, U.S.A. All ASWs were detected at a mean concentration that ranged from 0.13 (aspartame) to $29.4 \mu \mathrm{g} / \mathrm{L}$ (sucralose) in wastewater influent, 0.49 (aspartame) to $27.7 \mu \mathrm{g} / \mathrm{L}$ (sucralose) in primary influent, 0.11 (aspartame) to $29.6 \mu \mathrm{g} / \mathrm{L}$ (sucralose) in effluent, and from 0.08 (aspartame) to $0.65 \mu \mathrm{g} / \mathrm{g} \mathrm{dw}$ (sucralose) in sludge. Aspartame was found in $92 \%$ of influent SPM samples at a mean concentration of 444

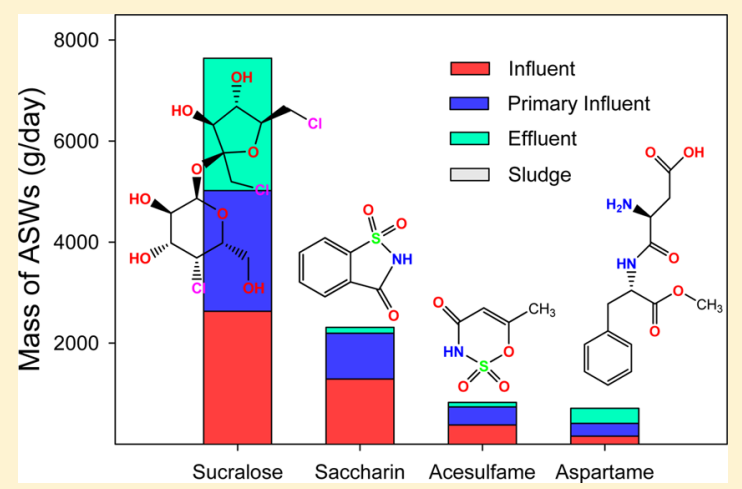
$\mathrm{ng} / \mathrm{g} \mathrm{dw}$, followed by acesulfame (92 ng/g) and saccharin (49 ng/g).

The fraction of the total mass of ASWs sorbed to SPM was in the rank order: aspartame $(50.4 \%)>$ acesulfame $(10.9 \%)>$ saccharin and sucralose (0.8\%). The sorption coefficients of ASWs ranged from 4.10 (saccharin) to $4540 \mathrm{~L} / \mathrm{kg}$ (aspartame). Significant removal of aspartame (68.2\%) and saccharin (90.3\%) was found in WWTPs; however, sucralose and acesulfame were less efficiently removed $(<2.0 \%)$. The total mass loading of sucralose, saccharin, and acesulfame in the WWTP that served a smaller population $(\sim 15000)$ was $1.3-1.5$ times lower than that in another WWTP that served a larger population $(\sim 100000)$. The average daily loading of sucralose in both WWTPs $(18.5 \mathrm{~g} / \mathrm{d} / 1000$ people $)$ was $\sim 2$ times higher than the average loading of saccharin. The daily discharge of sucralose from the WWTPs was the highest $(17.6 \mathrm{~g} / \mathrm{d} / 1000$ people $)$, followed by acesulfame $(1.22 \mathrm{~g} / \mathrm{d} / 1000$ people $)$, and saccharin $(1.07 \mathrm{~g} / \mathrm{d} / 1000$ people $)$. Approximately, $1180 \mathrm{~g}$ of saccharin and $291 \mathrm{~g}$ of acesulfame were transformed in or removed daily from the two WWTPs. This is the first study to describe the fate of ASWs, including the fraction found in SPM and in sludge, in addition to the aqueous portion of wastewater in WWTPs.

\section{INTRODUCTION}

Artificial sweeteners (ASWs) are used as substitutes for sugar in food additives, health and dietary products (foods and beverages), and animal feeds. ${ }^{1}$ Sucralose, saccharin, aspartame, neotame, and acesulfame are "low-calorie sweeteners" currently approved for use in the U.S.A. ${ }^{2}$ Sucralose was approved for use as an additive in over 4000 food products in $>80$ countries, ${ }^{3}$ with annual sales in the U.S.A. at $>\$ 170$ million in $2004{ }^{4,5}$ The global consumption of acesulfame and saccharin in 2005 was 4 and 37 thousand metric tons, respectively. ${ }^{6}$

ASWs are excreted mostly unchanged from the human body, flow down the drain, and are discharged into the environment through wastewater treatment plants (WWTPs). ${ }^{7,8}$ Hydrophilic ASWs $\left(\right.$ solubility $=565-9.1 \times 10^{5} \mathrm{mg} / \mathrm{L}$ at $\left.25^{\circ} \mathrm{C}\right)($ Table 1$)$ are not efficiently removed through wastewater treatment processes. For instance, removal efficiencies for acesulfame and sucralose in WWTPs were reported to be $<20 \% .^{9-12}$ As a consequence, ASWs have been reported to occur at ng/L to $\mathrm{mg} / \mathrm{L}$ levels in wastewaters, ${ }^{13}$ river and coastal waters, ${ }^{4,10}$ landfill leachates, ${ }^{14}$ and drinking water. ${ }^{15,16}$ Thus far, only four reports have shown the occurrence of ASWs in sewage sludge; ${ }^{12,17-19}$ however, no previous studies have examined the occurrence of ASWs in suspended particulate matter (SPM) of wastewater.

Sucralose and acesulfame are persistent in the environment. Acesulfame was found at similar concentrations in a septic tank and a $\sim 15$-year-old septic plume. ${ }^{20}$ Due to their ubiquitous occurrence and persistence, sucralose ${ }^{5,15,21}$ and acesulfame $e^{9,20}$ were used as tracers of wastewater contamination in groundwater, landfill leachate, ${ }^{14}$ and drinking water. However, longterm consequences of ubiquitous distribution of ASWs in aquatic ecosystems and their potential transformations are poorly characterized. ${ }^{7}$ ASWs in the aquatic environment have

Received: September 29, 2014

Revised: October 27, 2014

Accepted: November 3, 2014 
Table 1. Physiochemical Properties of Select ASWs (Predicted Data from www.chemspider.com, Based on USEPA's EPISuite) ${ }^{17}$

\begin{tabular}{|c|c|c|c|c|c|c|c|c|}
\hline analytes & CAS\# & $\log K_{\mathrm{ow}}{ }^{a}$ & $\log K_{\mathrm{oc}}^{b}$ & $\begin{array}{c}\text { solubility }{ }^{c}(\mathrm{mg} / \mathrm{L}) \\
\text { at } 25{ }^{\circ} \mathrm{C}\end{array}$ & $\begin{array}{c}\text { removal }^{d} \\
\text { in WWTP (\%) }\end{array}$ & $\begin{array}{l}\text { adsorption }^{d} \\
\text { on sludge (\%) }\end{array}$ & biodegradation $^{d}(\%)$ & $\begin{array}{c}\text { half-life }^{e} \\
\text { in sediment }(h)\end{array}$ \\
\hline sucralose & $56038-13-2$ & -1.00 & 1.00 & $2.75 \times 10^{4}$ & 1.50 & 1.75 & 0.09 & $8.10 \times 10^{3}$ \\
\hline aspartame & $22839-47-0$ & 0.07 & 1.786 & 564.7 & 1.85 & 1.76 & 0.09 & $3.24 \times 10^{3}$ \\
\hline saccharin & $81-07-2$ & 0.45 & 1.507 & 789.2 & 1.88 & 1.79 & 0.09 & $3.24 \times 10^{3}$ \\
\hline acesulfame & $33665-90-6$ & -1.33 & 1.302 & $9.1 \times 10^{5}$ & 1.85 & 1.75 & 0.09 & $3.24 \times 10^{3}$ \\
\hline
\end{tabular}

${ }^{a}$ Based on KOWWIN v1.67 estimate. ${ }^{b}$ Based on PCKOCWIN v1.66. ${ }^{c}$ Based on WSKOW V1.41. ${ }^{d}$ Based on STPWINTM. ${ }^{e}$ Based on level-III fugacity model via WOVWIN.

been shown to alter the physiology and locomotion of Daphnia magna, a planktonic crustacean at $\leq 0.5 \mu \mathrm{g} / \mathrm{L} .{ }^{22}$ ASWs also can interfere with photosynthesis in plants. ${ }^{23}$ Acesulfame is a precursor for the formation of several oxidation byproducts during advanced wastewater or drinking water treatment processes, such as ozonation. ${ }^{24}$

The primary goal of this study was to determine the fate of sucralose, acesulfame, saccharin, and aspartame in WWTPs. ASWs were analyzed in wastewater influent, primary effluent, final effluent, SPM, and treated sludge from two WWTPs in the Albany area of New York State. This is the first study to describe the fate of ASWs, including the fraction in SPM and in sludge, in addition to the aqueous portion of wastewater in WWTPs. The fraction of each of the ASWs sorbed to SPM was calculated and utilized in the estimation of mass loadings and environmental emissions of these chemicals from WWTPs. The sorption coefficients and removal efficiencies of ASWs through wastewater treatment processes also were calculated.

\section{MATERIALS AND METHODS}

Reagents and Chemicals. Analytical standards of saccharin and sucralose were purchased from Sigma-Aldrich (St. Louis, MO). Acesulfame potassium and aspartame were purchased from Supelco (Bellefonte, PA). Saccharin $-{ }^{13} C_{6}$, sucralose- $\mathrm{D}_{6}$, acesulfame- $\mathrm{D}_{4}$, and aspartame- $\mathrm{D}_{5}$ were purchased from Santa Cruz Biotechnology, Inc. (Dallas, TX). ${ }^{17}$ Purity of all of the standards was $\geq 98 \%$. All organic solvents (HPLC grade) and ammonium hydroxide (29.5\% as ammonia) were purchased from Mallinckrodt Baker (Phillipsburg, NJ). Ultrapure water was prepared using the Barnstead International's ultrapure system (Dubuque, IA). All standard stock solutions were stored at $-20{ }^{\circ} \mathrm{C}$.

Sample Collection and Preparation. Detailed information about the collection of samples from two WWTPs is provided elsewhere. ${ }^{25}$ Briefly, 24-h composite wastewater samples, including raw wastewater (influent), primary-treated wastewater (primary effluent), secondary-treated wastewater (effluent or final effluent), and sludge were collected consecutively over a seven-day period, from July 12 to 18 , 2013, from two WWTPs in the Albany area of New York State, U.S.A. The two plants are denoted as $\mathrm{WWTP}_{\mathrm{A}}$ (population served $\sim 15000$ ) and $\mathrm{WWTP}_{\mathrm{B}}$ (population served $\sim 100000$ ), with a treatment capacity of 9.5 and 132 MLD [million liters per day], respectively. Both WWTPs used activated biological treatment. The activated sludge $(3.9 \%$ solid, determined gravimetrically) samples were collected for 7 days from $\mathrm{WWTP}_{\mathrm{A}}$ and four consecutive days within the sampling week from $\mathrm{WWTP}_{\mathrm{B}}$. The activated sludge samples from both WWTPs were the combined sludge produced after primary and secondary treatments. All samples were collected in certified precleaned amber glass jars with Teflon-faced caps, shipped to the laboratory, and stored in a refrigerator at $4{ }^{\circ} \mathrm{C}$ until extraction.

The procedure for the extraction of wastewater, SPM, and sludge has been described elsewhere. ${ }^{17,25}$ The wastewater samples $(100 \mathrm{~mL})$ were centrifuged at $5000 \mathrm{~g}$ for $10 \mathrm{~min}$, and the supernatant was filtered through a glass fiber filter $(37 \mathrm{~mm}$, pore size $1 \mu \mathrm{m}$; GE Osmonics Inc., Minnetonka, MN) to separate SPM. Wastewater samples $(100 \mathrm{~mL})$ were spiked with a mixture of labeled internal standards of the target artificial sweeteners ( 25 or $50 \mathrm{ng}$ ) prior to extraction. The aqueous samples were extracted by passage through Oasis HLB $6 \mathrm{~cm}^{3}$ (200 mg; Waters, Milford, MA) solid phase extraction (SPE) cartridges. Prior to use, the cartridges were conditioned with 5 $\mathrm{mL}$ of methanol and $5 \mathrm{~mL}$ of milli-Q water, and wastewater samples were loaded at $\sim 1 \mathrm{~mL} / \mathrm{min}$. Cartridges were allowed to dry for $\sim 30$ min under vacuum and then eluted with $6 \mathrm{~mL}$ of methanol followed by $3 \mathrm{~mL}$ of a mixture of acetone, methanol, and ethyl acetate $(2: 2: 1 \mathrm{v} / \mathrm{v} / \mathrm{v})$. Cartridges also were eluted with $3 \mathrm{~mL}$ of methanol containing 5\% ammonia. The eluents were combined and concentrated to $\sim 100 \mu \mathrm{L}$ under a gentle stream of nitrogen at $35{ }^{\circ} \mathrm{C}$ using a TurboVap Evaporator (Zymark, Inc., Hopkinton, MA).

Similarly, $\sim 0.1 \mathrm{~g}$ of freeze-dried sludge was spiked with a mixture of internal standards ( 25 or $50 \mathrm{ng}$ ) prior to extraction. Spiked sludge samples were vortex-mixed for $1 \mathrm{~min}$ and extracted with $6 \mathrm{~mL}$ of methanol/water mixture $(5: 3 \mathrm{v} / \mathrm{v})$ using an ultrasonic bath (Branson Ultrasonics 3510R-DTH; Danbury, CT) for $30 \mathrm{~min}$. Extracts were centrifuged at $4500 \mathrm{~g}$ for 5 min (Eppendorf Centrifuge 5804, Hamburg, Germany), supernatant was collected in a polypropylene tube, and the extraction was repeated twice. The extracts were combined and concentrated to $\sim 1 \mathrm{~mL}$ under a gentle stream of nitrogen. The concentrated extract was diluted with ultrapure water to $\sim 6 \mathrm{~mL}$ and purified by passage through SPE cartridges, as described above. The entire contents of SPM in the preweighed glassfiber filter (obtained from centrifugation and filtration of 100 $\mathrm{mL}$ of influents, primary effluents, and effluents) were transferred into a preweighed polypropylene tube, freezedried, spiked with the internal standards, and extracted as described above for sludge. The final volume of the extract was $1 \mathrm{~mL}$, and $10 \mu \mathrm{L}$ of the extract was injected into HPLC-MS/ MS.

Instrumental Analysis. Detailed information on instrumental analysis, including MS/MS transitions, analyte peak identification, quantification, and quality assurance and quality control (QA/QC) protocols, has been provided elsewhere. ${ }^{17}$ The continuing calibration verification standards injected before and after every batch $(n=21)$ of samples analyzed showed recoveries at $100 \pm 25 \%$. The regression coefficients $\left(r^{2}\right)$ for 7- to 10-point calibration standards calculated by equal weighting quadratic regression were $\geq 0.99$ for all target analytes. A method blank was analyzed with every batch of 
samples. The measured concentrations of target analytes in procedural blanks were below the corresponding limit of quantitation (LOQ). The concentrations of target chemicals in SPM and sludge are reported on a dry-weight basis, unless stated otherwise.

One sample was selected randomly for matrix spike (MS) and matrix spike duplicate (MSD) analyses with each batch of samples analyzed. Target analytes and corresponding internal standards were each spiked at 25 and $50 \mathrm{ng}$, respectively, and were passed through the entire analytical procedure. The overall average recoveries of artificial sweeteners in spiked matrices were $66 \pm 17 \%, 68 \pm 13 \%$, and $122 \pm 10 \%$ for wastewater, sludge, and SPM, respectively.

Calculations. The fraction of the total mass of ASWs sorbed to SPM, removal efficiency of ASWs through the treatment processes, mass loadings of ASWs in WWTPs, and environmental emission of ASWs from WWTPs were calculated using the following equations (eqs 1-4), as reported by Subedi and Kannan (2014). ${ }^{25}$

$$
\begin{aligned}
& P_{\mathrm{SPM}}=\frac{\left(\frac{C_{\mathrm{SPM}} \times M_{\mathrm{SPM}}}{V_{\mathrm{w}}}\right)}{\left(\frac{C_{\mathrm{SPM}} \times M_{\mathrm{SPM}}}{V_{\mathrm{W}}}\right)+C_{\mathrm{W}}} \times 100 \\
& \mathrm{RE}(\%)=\frac{\left(\frac{100 \times C_{\mathrm{i}}}{100-P_{\mathrm{SPMi}}}\right)-\left(\frac{100 \times C_{\mathrm{e}}}{100-P_{\mathrm{SPMe}}}\right)}{\left(\frac{100 \times C_{\mathrm{i}}}{100-P_{\mathrm{SPMi}}}\right)} \times 100 \\
& \mathrm{ML} / 1000 \text { people }=C_{\mathrm{i}} \times F \times\left(\frac{100}{100-P_{\mathrm{SPM}}}\right) \times\left(\frac{1}{\text { pop. }}\right) \\
& \mathrm{E} / 1000 \text { people }=\left[\left(C_{\mathrm{E}} \times F\right)\left(\frac{100}{100-P_{\mathrm{SPM}}}\right)\right. \\
& \left.+\left(C_{\mathrm{S}} \times \mathrm{TSP}\right)\right]\left(\frac{1000}{\text { pop. }}\right)\left(\frac{1}{10^{6}}\right)
\end{aligned}
$$

where $P_{\mathrm{SPM}}$ is the fraction of the total mass of ASWs sorbed to SPM (\%); $C_{\text {SPM }}$ is the concentration of ASWs in SPM (ng/g); $M_{\mathrm{SPM}}$ is the mass of SPM analyzed $(\mathrm{g}) ; V_{\mathrm{W}}$ is the volume of wastewater (L) used to obtain $M_{\mathrm{SPM}} ; C_{\mathrm{W}}$ is the concentration of ASWs in wastewater $(\mathrm{ng} / \mathrm{L})$; RE is the removal efficiency; $C_{\mathrm{i}}$ is the concentration of ASWs in wastewater influent $(\mathrm{ng} / \mathrm{L}) ; C_{\mathrm{e}}$ is the concentration of ASWs in wastewater effluent $(\mathrm{ng} / \mathrm{L}) ; P_{\mathrm{SPMi}}$ is the fraction of the total load of ASWs sorbed to SPM (\%) in wastewater influent; $P_{\mathrm{SPMe}}$ is the fraction of the total load of ASWs sorbed to SPM (\%) in wastewater effluent; ML is the mass load, an estimated amount of individual ASW introduced into a WWTP per day per 1000 people served $(\mathrm{g} / \mathrm{d} / 1000$ people); $F$ is the daily flow of wastewater influent $(\mathrm{L} / \mathrm{d})$ over a 24-h period; $C_{s}$ is the concentration of ASWs in sludge $(\mathrm{ng} / \mathrm{g}$ wet weight); pop. is the population; TSP is the total sludge production ( $\mathrm{g} / \mathrm{d}$ wet weight); population is the number of people served by the WWTP; and E/1000 people is the emission quantity of ASWs discharged through wastewater effluent, SPM, and sludge ( $\mathrm{mg} / \mathrm{d} / 1000$ people).

\section{RESULTS AND DISCUSSION}

Artificial Sweeteners in Wastewater. All four ASWs were found in all wastewater influent samples (df: 100\%) from both WWTPs (Table 2). Sucralose was found at the highest mean concentration in wastewater influent, at 33.0 and 25.9 $\mu \mathrm{g} / \mathrm{L}$ in $\mathrm{WWTP}_{\mathrm{A}}$ and $\mathrm{WWTP}_{\mathrm{B}}$, respectively. The mean concentrations of sucralose in influent were approximately 2 , 28 , and 257 times higher than the mean concentrations of saccharin, acesulfame, and aspartame, respectively. It has been reported that acesulfame and saccharin were the predominant ASWs, followed by sucralose in WWTPs in Germany, ${ }^{11}$ a pattern different from that found in our study.

The measured concentration of sucralose was $\sim 2$ times higher than the concentration previously estimated for WWTP influents in the U.S.A. ${ }^{5}$ and at least an order of magnitude higher than that reported in another study $(1.38 \mu \mathrm{g} / \mathrm{L})$ from the U.S.A. ${ }^{26}$ The measured concentration of sucralose in influent was similar to that reported for influent from two WWTPs in $\mathrm{Canada}^{27}$ and $\sim 30$ times higher than that reported in Germany. ${ }^{11}$

The mean concentration of saccharin in influent from two WWTPs $(16.4 \mu \mathrm{g} / \mathrm{L})$, in this study, was similar to that reported from Spain ${ }^{18}$ and $\sim 2$ times higher than that found in China ${ }^{10}$ and Germany. ${ }^{11}$ Nevertheless, the mean concentration of acesulfame in influent $(1.08 \mu \mathrm{g} / \mathrm{L})$ was $\sim 25$ and 40 times lower than that reported from Spain ${ }^{18}$ and Germany, ${ }^{11}$ respectively.

Aspartame has never been measured in wastewater samples from the U.S.A.; the mean concentration of aspartame determined in influent in this study $(0.13 \mu \mathrm{g} / \mathrm{L})$ was 2 times higher than that reported from China ${ }^{10}$ and up to an order of magnitude lower than that reported in Singapore. ${ }^{28}$ Aspartame was reported to occur (up to $11.5 \mathrm{ng} / \mathrm{L}$ ) in WWTP effluents in Switzerland. ${ }^{11,29}$

Artificial Sweeteners in Sludge. Thus, far, only four studies have determined ASWs in sewage sludge. ${ }^{12,17-19}$ This is the first report of the occurrence of ASWs in sewage sludge from the U.S.A. ASWs are hydrophilic compounds and partition primarily to the aqueous phase (water solubilities range from 565 to $1.00 \times 10^{6} \mathrm{mg} / \mathrm{L}$ at $25^{\circ} \mathrm{C}$ ) (Table 1). High levels of ASWs found in sludge, despite their relatively low sorption coefficients, can be due to high loadings into the WWTPs. However, in mass loading calculations, omission of the fraction of the environmental contaminants that partition into the particulate fraction, including sewage sludge, can underestimate the actual loadings into the WWTPs. ${ }^{25}$

Sucralose (df: $55 \%)$, saccharin (df: $73 \%$ ), acesulfame (df: $100 \%$ ), and aspartame (df: 93\%) were found in the sludge samples collected from both WWTPs at a mean concentration that ranged from 118 (acesulfame) to $506 \mathrm{ng} / \mathrm{g} \mathrm{dw}$ (sucralose) (Table 2). The mean concentrations of ASWs measured in this study were similar to those reported in Spain. ${ }^{18}$ Nevertheless, mean concentration of sucralose in sludge in our study was $\sim 45$ times higher than that reported in WWTPs from Sweden. ${ }^{12}$

The concentrations of ASWs measured in sludge were compared with those reported from Korea (Figure 1). ${ }^{17}$ The rank order of mean concentrations of ASWs in sludge from Korea was as follows: aspartame $>$ saccharin $>$ acesulfame > sucralose, whereas that in the U.S.A. was as follows: sucralose > aspartame $\approx$ saccharin $>$ acesulfame. The concentration of sucralose in sludge from the U.S.A. was an order of magnitude higher than that found in Korea; however, the concentrations of aspartame and saccharin in Korea were 2-3 times higher than those found in the U.S.A. The differences in the patterns of ASWs in sludge in the U.S.A. and Korea suggest differences in per-capita consumption of individual ASWs and the removal in WWTP treatment processes. Sucralose is the most frequently 

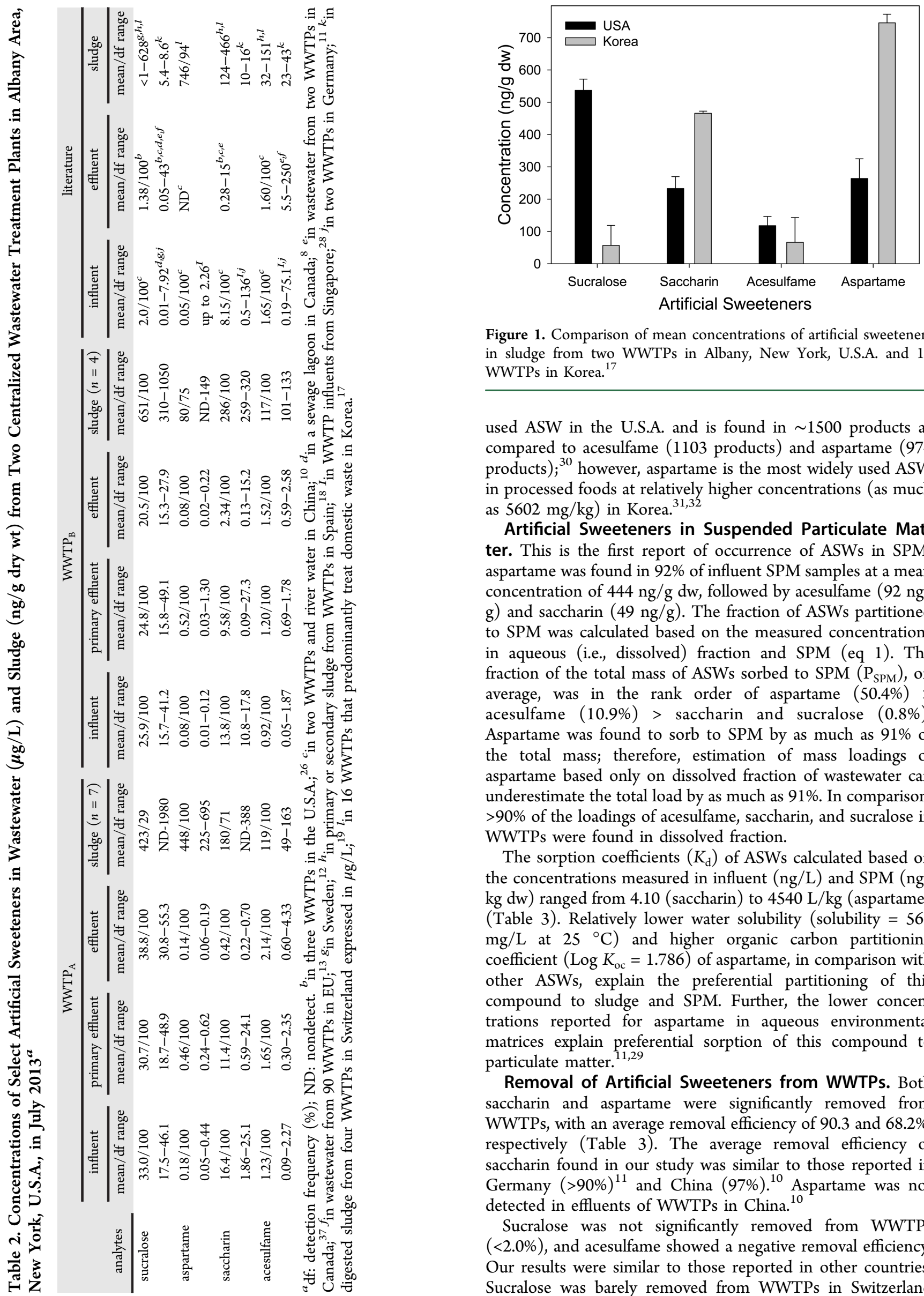

Figure 1. Comparison of mean concentrations of artificial sweeteners in sludge from two WWTPs in Albany, New York, U.S.A. and 16 WWTPs in Korea. ${ }^{17}$

used ASW in the U.S.A. and is found in $\sim 1500$ products as compared to acesulfame (1103 products) and aspartame (974 products); ${ }^{30}$ however, aspartame is the most widely used ASW in processed foods at relatively higher concentrations (as much as $5602 \mathrm{mg} / \mathrm{kg}$ ) in Korea. ${ }^{31,32}$

Artificial Sweeteners in Suspended Particulate Matter. This is the first report of occurrence of ASWs in SPM; aspartame was found in $92 \%$ of influent SPM samples at a mean concentration of $444 \mathrm{ng} / \mathrm{g} \mathrm{dw}$, followed by acesulfame ( $92 \mathrm{ng} /$ g) and saccharin $(49 \mathrm{ng} / \mathrm{g})$. The fraction of ASWs partitioned to SPM was calculated based on the measured concentrations in aqueous (i.e., dissolved) fraction and SPM (eq 1). The fraction of the total mass of ASWs sorbed to SPM $\left(\mathrm{P}_{\mathrm{SPM}}\right)$, on average, was in the rank order of aspartame (50.4\%) > acesulfame $(10.9 \%)>$ saccharin and sucralose $(0.8 \%)$. Aspartame was found to sorb to SPM by as much as $91 \%$ of the total mass; therefore, estimation of mass loadings of aspartame based only on dissolved fraction of wastewater can underestimate the total load by as much as $91 \%$. In comparison, $>90 \%$ of the loadings of acesulfame, saccharin, and sucralose in WWTPs were found in dissolved fraction.

The sorption coefficients $\left(K_{\mathrm{d}}\right)$ of ASWs calculated based on the concentrations measured in influent (ng/L) and SPM (ng/ $\mathrm{kg} \mathrm{dw}$ ) ranged from 4.10 (saccharin) to $4540 \mathrm{~L} / \mathrm{kg}$ (aspartame) (Table 3). Relatively lower water solubility (solubility $=565$ $\mathrm{mg} / \mathrm{L}$ at $25{ }^{\circ} \mathrm{C}$ ) and higher organic carbon partitioning coefficient $\left(\log K_{\mathrm{oc}}=1.786\right)$ of aspartame, in comparison with other ASWs, explain the preferential partitioning of this compound to sludge and SPM. Further, the lower concentrations reported for aspartame in aqueous environmental matrices explain preferential sorption of this compound to particulate matter. ${ }^{11,29}$

Removal of Artificial Sweeteners from WWTPs. Both saccharin and aspartame were significantly removed from WWTPs, with an average removal efficiency of 90.3 and $68.2 \%$, respectively (Table 3 ). The average removal efficiency of saccharin found in our study was similar to those reported in Germany $(>90 \%)^{11}$ and China $(97 \%) .{ }^{10}$ Aspartame was not detected in effluents of WWTPs in China. ${ }^{10}$

Sucralose was not significantly removed from WWTPs $(<2.0 \%)$, and acesulfame showed a negative removal efficiency. Our results were similar to those reported in other countries. Sucralose was barely removed from WWTPs in Switzerland 
Table 3. Mass Load, Environmental Emission, Sorption Coefficient, And an Average Removal Efficiency of Select Artificial Sweeteners through WWTPs in Albany Area, New York, U.S.A.

\begin{tabular}{|c|c|c|c|c|c|c|}
\hline \multirow[b]{2}{*}{ analyte } & \multicolumn{2}{|c|}{ mass load (g/d/1000 people) } & \multicolumn{2}{|c|}{$\begin{array}{c}\text { discharge via WWTPs }(\mathrm{g} / \mathrm{d} / 1000 \\
\text { people) }\end{array}$} & \multirow[b]{2}{*}{$K_{\mathrm{d}}(\mathrm{L} / \mathrm{kg})$} & \multirow[b]{2}{*}{ average $\%$ removal } \\
\hline & $\mathrm{WWTP}_{\mathrm{A}}$ & WWTP $_{\mathrm{B}}$ & $\mathrm{WWTP}_{\mathrm{A}}$ & WWTP $_{\mathrm{B}}$ & & \\
\hline sucralose & 15.4 & 21.6 & 18.1 & 17.1 & 5.10 & 1.60 \\
\hline aspartame & 0.47 & 0.21 & 0.12 & 0.08 & 4540 & 68.2 \\
\hline saccharin & 7.68 & 11.5 & 0.20 & 1.95 & 4.10 & 90.3 \\
\hline acesulfame & 0.70 & 0.89 & 1.10 & 1.35 & 289 & -54.2 \\
\hline
\end{tabular}

$(-5 \%),{ }^{9}$ China $(17.7 \%),{ }^{10}$ Germany $(20 \%),{ }^{11}$ and Sweden $(<10 \%) .{ }^{12}$ Sucralose is not liable for microbial degradation due to the presence of chlorine atoms. ${ }^{7,26}$ Nevertheless, sucralose was used as a molecular marker of wastewater contamination in groundwater and drinking water. $5,15,21$ Similarly, negative removal efficiency of acesulfame was reported in municipal WWTPs in Switzerland (-9\%); ${ }^{9}$ however, no clear explanation is available to support the negative removal of acesulfame from WWTPs.

Mass loads of Artificial Sweeteners in WWTPs. The total mass of ASWs discharged into the WWTP was estimated based on the measured concentrations in influents, daily flow of influent, and the population served by the WWTP (eq 3). The total loadings of sucralose, saccharin, and acesulfame into $\mathrm{WWTP}_{\mathrm{A}}$, which serves a smaller population $(\sim 15000)$ were 1.3-1.5 times lower than those of $\mathrm{WWTP}_{\mathrm{B}}$, which serves a larger population $(\sim 100000)$; however, the total loadings of aspartame into $\mathrm{WWTP}_{\mathrm{A}}$ were 2.3 times higher than those of $\mathrm{WWTP}_{\mathrm{B}}$ (Table 3). The average loadings of sucralose into both WWTPs $(18.5 \mathrm{~g} / \mathrm{d} / 1000$ people) were $\sim 2$ times higher than the loadings of saccharin. The average loadings of sucralose in two WWTPs in our study (U.S.A.) were $\sim 12$ and 100 times higher than those reported in Switzerland (Canton of Zurich, average population $=58840$ ) and Germany (EggensteinLeopoldshafen and Karlsruhe, average population $=185000)$, respectively. However, the average loadings of acesulfame in our WWTPs (U.S.A.) were $\sim 13$ and 15 times lower than those reported in Switzerland and Germany, respectively (Figure 2). ${ }^{9,11}$ High consumption and a broad-range application of sucralose in the U.S.A. can explain the high concentration of

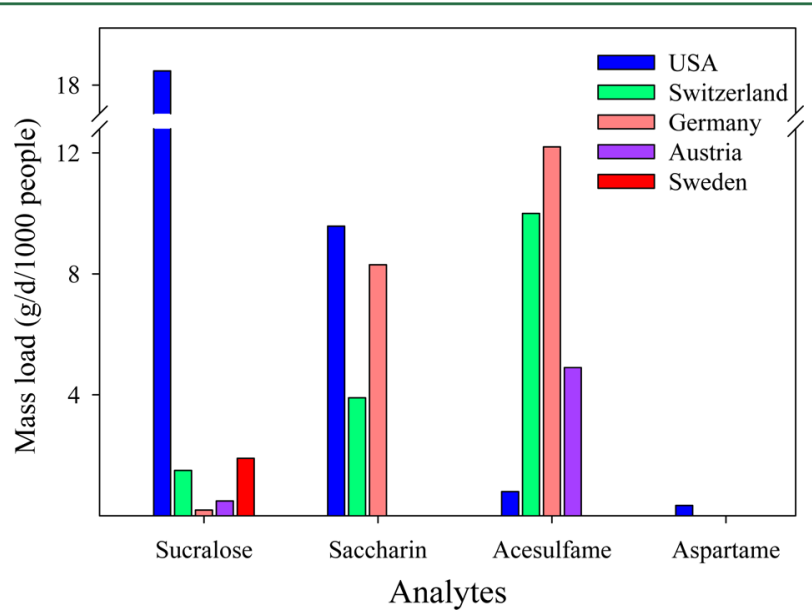

Figure 2. Average loadings of artificial sweeteners introduced into WWTP $(\mathrm{g} / \mathrm{d} / 1000$ people) in this study (Albany, New York, U.S.A.), and those reported in Switzerland, ${ }^{9}$ Germany, ${ }^{11,33}$ Austria, ${ }^{33}$ and Sweden. ${ }^{12}$ this compound in WWTPs. ${ }^{33}$ In general, studies from several European nations have showed the prevalence of acesulfame and saccharin, whereas those from the U.S.A. have showed the predominance of sucralose in WWTPs.

Environmental Emission of Artificial Sweeteners through WWTPs. The total mass of ASWs discharged through WWTP effluents and sewage sludge was calculated based on the concentrations measured in effluents, SPM, and sludge as well as by the daily flow rate of effluents, total sludge production rate, and the population served by the WWTPs (eq 4). Sucralose was found to be the most highly discharged (17.6 $\mathrm{g} / \mathrm{d} / 1000$ people) ASW from the WWTPs, followed by acesulfame and saccharin (Table 3 ). These amounts correspond to an estimated annual discharge of $624 \mathrm{~kg}$ of sucralose, $71.0 \mathrm{~kg}$ of saccharin, $2.90 \mathrm{~kg}$ of aspartame, and $49.1 \mathrm{~kg}$ of acesulfame from a WWTP that serves a population of 100000 (influent flow rate of $\left.83300 \mathrm{~m}^{3} / \mathrm{d}\right)$. Gan et al. $(2013)^{10}$ estimated an annual discharge of $26 \mathrm{~kg}$ of saccharin and $1600 \mathrm{~kg}$ of acesulfame from a WWTP (treatment capacity of $260000 \mathrm{~m}^{3}$ / d) in China.

There was no considerable removal of sucralose in the activated sludge treatment process; however, $\sim 1180 \mathrm{~g}$ of saccharin and $291 \mathrm{~g}$ of acesulfame were estimated to be transformed or removed daily from the WWTPs studied (Figure 3). Sucralose is not liable for microbial degradation due to the presence of chlorine atoms; however, heterocyclic saccharin can undergo ring opening and be transformed into other products. ${ }^{26}$ Recently, photolytic transformation byproducts of acesulfame, such as hydroxylated acesulfame, isoacesulfame, and amidosulfonic acid, have been identified; ${ }^{34}$ however, further investigation is required to identify and/or quantify the transformation byproducts of ASWs during wastewater treatments. Conversely, aspartame mass loading increased in the wastewater treatment processes. Unlike other ASWs, aspartame is almost completely metabolized in the human body ${ }^{35,36}$ and possibly excreted as a conjugate of glucuronide or sulfate. Wastewater treatment processes may deconjugate or transform the metabolites or conjugates into the parent molecule. Aspartame can lose its acetyl or amine group under different $\mathrm{pH}$ conditions during wastewater treatment. ${ }^{26}$

\section{CONCLUSIONS}

In summary, mass loadings, removal efficiencies, and environmental emission of sucralose, saccharin, aspartame, and acesulfame were determined, based on the concentrations measured in wastewater, SPM, and sludge, from two WWTPs in the Albany area of New York State, U.S.A. The fraction of the total mass of ASWs sorbed to SPM was in the rank order: aspartame $(50.4 \%)>$ acesulfame $(10.9 \%)>$ saccharin and sucralose $(0.8 \%)$. This study reports aspartame in wastewater samples for the first time in the U.S.A. Aspartame and saccharin were significantly removed from WWTPs; however, sucralose 


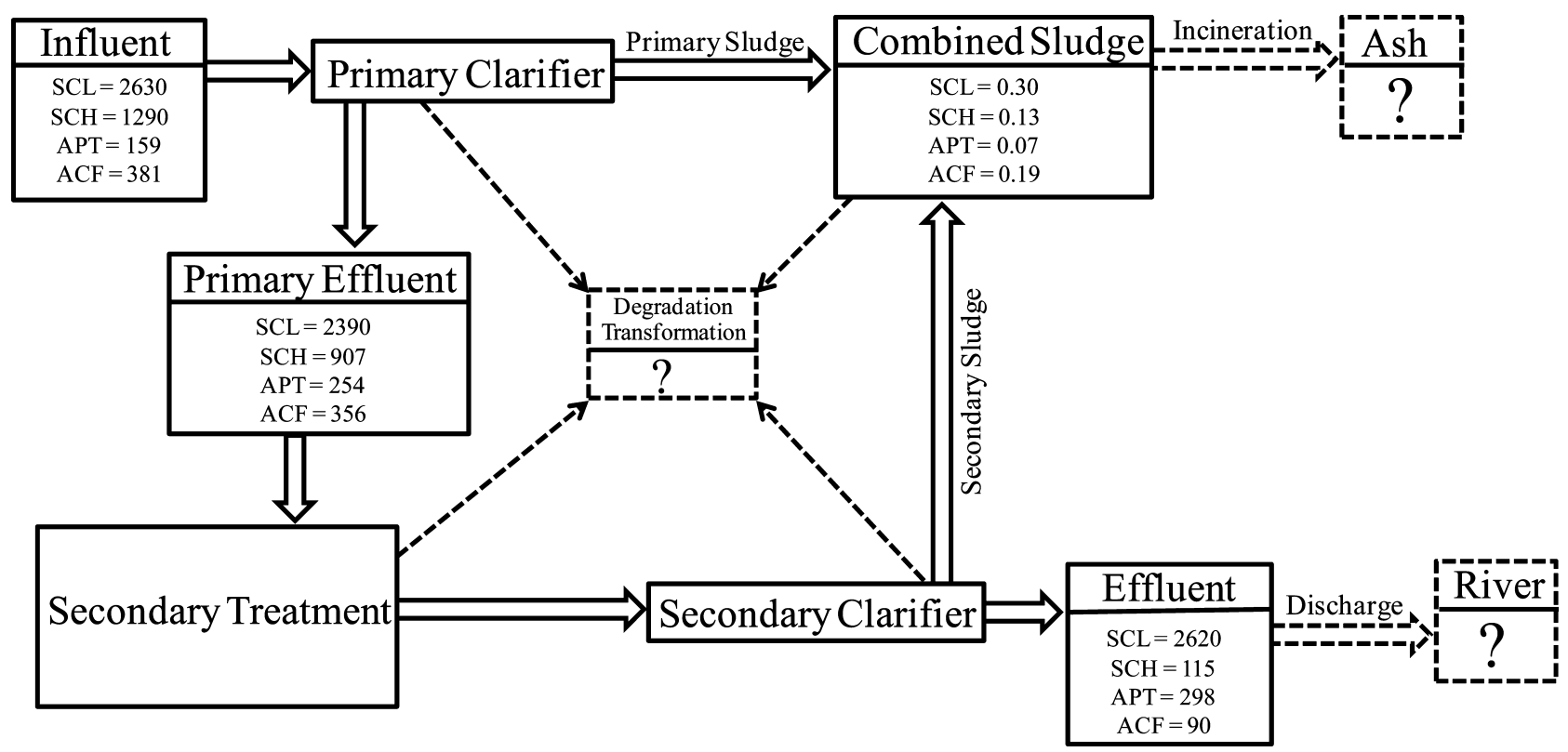

Figure 3. A schematic diagram showing the fate of select artificial sweeteners through a WWTP serving a population of $\sim 100000$ in Albany area, New York, U.S.A. The total amount $(\mathrm{g})$ of artificial sweeteners discharged daily at different stages of wastewater treatment is shown; sucralose (SCL), saccharin $(\mathrm{SCH})$, aspartame (APT), and acesulfame (ACF).

and acesulfame were removed at $<2.0 \%$. The average daily loading of sucralose in both WWTPs was $\sim 2$ times higher than the average loadings of saccharin; consequently, the discharge of sucralose from WWTPs was found highest among the ASWs studied. Saccharin ( $\sim 1180 \mathrm{~g} /$ day) and acesulfame $(291 \mathrm{~g} /$ day $)$ were transformed in or removed daily from the two WWTPs. Further investigation is required to identify and/or quantify the transformation products of ASWs in the wastewater treatment.

\section{AUTHOR INFORMATION}

\section{Corresponding Author}

*Phone: 1-518-474-0015; fax: 1-518-473-2895; e-mail: kurunthachalam.kannan@health.ny.gov.

\section{Notes}

The authors declare no competing financial interest.

\section{ACKNOWLEDGMENTS}

The authors would like to thank the WWTP facilities, Mr. Anthony DeJulio, and Mr. Jingchuan Xue for assistance with the sample collection.

\section{REFERENCES}

(1) Kroger, M.; Meister, K.; Kava, R. Low-calorie sweeteners and other sugar substitutes: A review of the safety issues. Comp. Rev. Food Sci. Food Safety 2006, 5, 35-47.

(2) FDA, FDA Consumer Management 2006; 40: 27-28.

(3) Torres, C. I.; Ramakrishna, S.; Chiu, C.; Nelson, K. G.; Westerhoff, P.; Krajmalnik-Brown, R. Fate of Sucralose During Wastewater Treatment. Environ. Eng. Sci. 2011, 28 (5), 325-331.

(4) Mead, R. N.; Morgan, J. B.; Avery, G. B.; Kieber, R. J.; Kirk, A. M.; Skrabal, S. A.; Willey, J. D. Occurrence of the artificial sweetener sucralose in coastal and marine waters of the United States. Chemosphere 2009, 116, 13-17.

(5) Soh, L.; Connors, K. A.; Brooks, B. W.; Zimmerman, J. Fate of sucralose through environmental and water treatment processes and impact on plant indicator species. Environ. Sci. Technol. 2011, 45 (4), 1363-1369.
(6) ISO, (International Sugar Organization). Sugar Substitutes: Recent Developments and Outlook; International Sugar Organization: London. 2008.

(7) Sang, Z.; Jiang, Y.; Tsoi, Y. K.; Leung, K. S. Evaluating the environmental impact of artificial sweeteners: a study of their distributions, photodegradation and toxicities. Water Res. 2014, 52, 260-274.

(8) Hoque, M. E.; Cloutier, F.; Arcieri, C.; McInnes, M.; Sultana, T.; Murray, C.; Vanrolleghem, P. A.; Metcalfe, C. D. Removal of selected pharmaceuticals, personal care products and artificial sweetener in an aerated sewage lagoon. Sci. Total Environ. 2014, 487, 801-812.

(9) Buerge, I. J.; Buser, H. R.; Kahle, M.; Muller, M. D.; Poiger, T. Ubiquitous occurrence of the artificial sweetener acesulfame in the aquatic environment: An ideal chemical marker of domestic wastewater in groundwater. Environ. Sci. Technol. 2009, 43 (12), 4381-4385.

(10) Gan, Z.; Sun, H.; Feng, B.; Wang, R.; Zhang, Y. Occurrence of seven artificial sweeteners in the aquatic environment and precipitation of Tianjin, China. Water Res. 2013, 47 (14), 4928-4937.

(11) Scheurer, M.; Brauch, H. J.; Lange, F. T. Analysis and occurrence of seven artificial sweeteners in German waste water and surface water and in soil aquifer treatment (SAT). Anal. Bioanal. Chem. 2009, 394 (6), 1585-1594.

(12) Lunden, E. B.; Scenson, A.; Viktor, T.; Woldeglorgis, A.; Remberger, M.; Kaj, L.; Dye, C.; Bjerke, A.; Schlabach, M., Measurements of sucralose in the Swedish Screening Program 2007-part I; Sucralose in surface waters and STP samples. Swedish Environmental Research Institute Ltd.: Stockholm.; 2008b. 2008.

(13) Loos, R.; Carvalho, R.; Antonio, D. C.; Comero, S.; Locoro, G.; Tavazzi, S.; Paracchini, B.; Ghiani, M.; Lettieri, T.; Blaha, L.; Jarosova, B.; Voorspoels, S.; Servaes, K.; Haglund, P.; Fick, J.; Lindberg, R. H.; Schwesig, D.; Gawlik, B. M. EU-wide monitoring survey on emerging polar organic contaminants in wastewater treatment plant effluents. Water Res. 2013, 47 (17), 6475-6487.

(14) Roy, J. W.; Van Stempvoort, D. R.; Bickerton, G. Artificial sweeteners as potential tracers of municipal landfill leachate. Environ. Pollut. 2014, 184, 89-93.

(15) Mawhinney, D. B.; Young, R. B.; Vanderford, B. J.; Borch, T.; Snyder, S. A. Artificial sweetener sucralose in U.S. drinking water systems. Environ. Sci. Technol. 2011, 45 (20), 8716-8722. 
(16) Scheurer, M.; Storck, F. R.; Brauch, H. J.; Lange, F. T. Performance of conventional multi-barrier drinking water treatment plants for the removal of four artificial sweeteners. Water Res. 2010, 44 (12), 3573-3584.

(17) Subedi, B.; Lee, S.; Moon, H. B.; Kannan, K. Emission of artificial sweeteners, select pharmaceuticals, and personal care products through sewage sludge from wastewater treatment plants in Korea. Environ. Int. 2014, 68, 33-40.

(18) Ordonez, E. Y.; Quintana, J. B.; Rodil, R.; Cela, R. Determination of artificial sweeteners in sewage sludge samples using pressurised liquid extraction and liquid chromatography-tandem mass spectrometry. J. Chromatogr. A 2013, 1320, 10-16.

(19) Buerge, I. J.; Keller, M.; Buser, H. R.; Muller, M. D.; Poiger, T. Saccharin and other artificial sweeteners in soils: estimated inputs from agriculture and households, degradation, and leaching to groundwater. Environ. Sci. Technol. 2011, 45 (2), 615-621.

(20) Robertson, W. D.; Stempvoort, D. R. V.; Solomon, D. K.; Homewood, J.; Brown, S. J.; Spoelstra, J.; Schiff, S. L. Persistence of artificial sweeteners in a 15 -year-old septic system plume. J. Hydrol. 2013, 477, 43-54.

(21) Oppenheimer, J.; Eaton, A.; Badruzzaman, M.; Haghani, A. W.; Jacangelo, J. G. Occurrence and suitability of sucralose as an indicator compound of wastewater loading to surface waters in urbanized regions. Water Res. 2011, 45 (13), 4019-4027.

(22) Wiklund, A. K.; Breitholtz, M.; Bengtsson, B. E.; AdolfssonErici, M. Sucralose-an ecotoxicological challenger? Chemosphere 2012, 86 (1), 50-55.

(23) Lubick, N. Artificial sweetener persists in the environment. Environ. Sci. Technol. 2008, 42 (9), 3125.

(24) Scheurer, M.; Godejohann, M.; Wick, A.; Happel, O.; Ternes, T. A.; Brauch, H. J.; Ruck, W. K.; Lange, F. T. Structural elucidation of main ozonation products of the artificial sweeteners cyclamate and acesulfame. Environ. Sci. Pollut. Res. Int. 2012, 19 (4), 1107-1118.

(25) Subedi, B.; Kannan, K. Mass loading and removal of select illicit drugs in two wastewater treatment plants in New York state and estimation of illicit drug usage in communities through wastewater analysis. Environ. Sci. Technol. 2014, 48 (12), 6661-6670.

(26) Ferrer, I.; Thurman, E. M. Analysis of sucralose and other sweeteners in water and beverage samples by liquid chromatography/ time-of-flight mass spectrometry. J. Chromatogr. A 2010, 1217 (25), 4127-4134.

(27) Stempvoort, D. R. V.; Roy, J. W.; Brown, S. J.; Bickerton, G. Artificial sweeteners as potential tracers in groundwater in urban environments. J. Hydrol. 2011, 104, 126-133.

(28) Tran, N. H.; Hu, J.; Ong, S. L. Simultaneous determination of PPCPs, EDCs, and artificial sweeteners in environmental water samples using a single-step SPE coupled with HPLC-MS/MS and isotope dilution. Talanta 2013, 113, 82-92.

(29) Berset, J. D.; Ochsenbein, N. Stability considerations of aspartame in the direct analysis of artificial sweeteners in water samples using high-performance liquid chromatography-tandem mass spectrometry (HPLC-MS/MS). Chemosphere 2012, 88 (5), 563-569.

(30) Yang, Q. Gain weight by "going diet?" Artificial sweeteners and the neurobiology of sugar cravings. Yale J. Biol. Med. 2010, 83, 101108.

(31) Ha, M.-S.; Ha, S.-D.; Choi, S.-H.; Bae, D.-H. Assessment of Korean consumer exposure to sodium saccharin, aspartame and stevioside. Food Addit. Contam. A 2013, 30 (7), 1238-1247.

(32) KFDA (Korean Food and Drugs Administration). Food \& Drug Statistical Yearbook. (In Korean), 2012.

(33) Lange, F. T.; Scheurer, M.; Brauch, H. J. Artificial sweetenersA recently recognized class of emerging environmental contaminants: A review. Anal. Bioanal. Chem. 2012, 403 (9), 2503-2518.

(34) Scheurer, M.; Schmutz, B.; Happel, O.; Brauch, H. J.; Wulser, R.; Storck, F. R. Transformation of the artificial sweetener acesulfame by UV light. Sci. Total Environ. 2014, 481, 425-432.

(35) Soffritti, M.; Padovani, M.; Tibaldi, E.; Falcioni, L.; Manservisi, F.; Belpoggi, F. The carcinogenic effects of aspartame: The urgent need for regulatory re-evaluation. Am. J. Ind. Med. 2014, 57 (4), 383397.

(36) Soffritti, M.; Belpoggi, F.; Degli Esposti, D.; Lambertini, L.; Tibaldi, E.; Rigano, A. First experimental demonstration of the multipotential carcinogenic effects of aspartame administered in the feed to Sprague-Dawley rats. Environ. Health Perspect. 2006, 114 (3), 379-385.

(37) Van Stempvoort, D. R.; Roy, J. W.; Grabuski, J.; Brown, S. J.; Bickerton, G.; Sverko, E. An artificial sweetener and pharmaceutical compounds as co-tracers of urban wastewater in groundwater. Sci. Total Environ. 2013, 461-462, 348-359. 